\title{
A Novel Frequency Communication Technology in Power Distribution Communication Network
}

\author{
Ying-Jun $\mathrm{LI}^{1, \mathrm{a}}$ \\ ${ }^{1}$ State Grid Shangrao Electric Power Supply Company, Shangrao Jiangxi, 334000, China
}

\begin{abstract}
With the expansion of the power terminal access network scale, the main road corridor resources, branch line cable Laying difficulties has become an important factor restricting the construction of the network. In this paper, we focus on the frequency communication technology in power distribution communication network, and design a novel technology in communication mode, error correcting coding and data transfer frame format. We also discuss the influence of voltage phase difference on power frequency communication. Meanwhile, we present the application scenario Electricity information collection, electricity remote control and other power business in smart grid with the novel frequency communication technology.
\end{abstract}

\section{Introduction}

As of December 2015, the distribution automation communication network of State Grid of China Company has deployed about 22.2 million power terminal devices, and covered about 28,000 lines of $10 \mathrm{kV}$ lines, about 300 million courts, about 12 million concentrators and other types of terminals and covered about 311 million smart energy meters[1]. The communication network met the basic business needs.

With the expansion of the power terminal access network scale, the main road corridor resources, branch line cable Laying difficulties has become an important factor restricting the construction of the network. Power line carrier communication (hereinafter referred to as: power line) And wireless communication with no wiring, covering a wide area, flexible networking and other characteristics, is to achieve the use of low power station Cost, wide coverage of the important means of communication access, optical fiber communication is an important complement and extension[2]. But head Before the power line and wireless communication two technical standards are relatively independent, failed to form an organic whole, there is coverage And network redundancy and other issues; at the same time in some remote areas is still a lack of effective means of communication, Letter blind area, the urgent need for effective communication to meet the basic needs of business, such as small hydropower / small thermal power station information collection, remote mountain meter reading and so on.

In this paper, we focus on the frequency communication technology in power distribution communication network, and design a novel technology in communication mode, error correcting coding and data transfer frame format. We also discuss the influence of voltage phase difference on

\footnotetext{
${ }^{a}$ Corresponding author: 1yj730@sina.com
} 
power frequency communication. Meanwhile, we present the application scenario Electricity information collection, electricity remote control and other power business in smart grid with the novel frequency communication technology.

The rest of this paper is organized as follows: in section 2, we present related works. Section 3 shows the system model including the communication model, the transmission framework, error correction and the influence of voltage phase difference on power frequency communication. And section 4 describes applications of new power frequency communication technology. Finally, we end the paper with a conclusion.

\section{Related works}

Foreign mainly in North America on the frequency of communications technology research, Mak, S.T. will supply lines, transformers and Load are equivalent to impedance, as a basis for the establishment of frequency communication signal modulation and transmission model, through simulation[3]. It is shown that when the modulation is made at 30 before the zero crossing of the voltage, the main component of the distortion signal is in the vicinity of the voltage zero to about $3 \mathrm{~ms}$, the frequency between $200 \mathrm{~Hz}$ to $600 \mathrm{~Hz}$, the amplitude of the distortion signal and the modulation transformer Capacity and modulation device parameters, when the distribution network load is heavy, the modulation signal will exist residual oscillation section[3,4].

The setting of the distortion signal and the threshold is related to the magnitude of the voltage distortion signal and the noise of the grid. Traditional upside Data demodulation is achieved by judging the difference between the positive and negative values of the current signal in the zero crossing of the voltage [5]. The receiver only set the detection window near the voltage zero crossing. Therefore, the reception of frequency communication includes synchronization detection and data solution Adjust the two links, the traditional synchronous detection method is based on the voltage zero-crossing time to determine whether the threshold Distortion signal to achieve, the downlink data demodulation is based on the power frequency cycle voltage zero-crossing time to change the situation[6].

In the frequency of communication signal transmission characteristics of the study, some simulation results show that the transmission frequency of the power frequency communication signal in China is close to that of the model simulation[7]. Meanwhile, the modulation signal is analyzed, and the modulation signal is analyzed by the field test and transmission simulation. The effect of residual oscillation on the signal detection is also proposed, and the method of reducing the residual oscillation signal by wavelet analysis is also proposed. Among them, there is a system voltage phase difference between the secondary winding wires of the Dy11 transformer, and the frequency attenuation is affected by the frequency communication signal crossing the type of transformer[ 7,8$]$.

\section{The novel frequency communication technology in power distribution network}

In order to make the power line frequency communication technology can reliably transmit data, as a smart grid construction feasible Information transmission options, this paper to carry out the new power line frequency communication technology research, including multi-feeder, Three-phase, multichannel signal transmission model research, multi-channel power frequency signal modulation and demodulation technology research, communication agreement research[9].

\subsection{Research model}

The protocol of the frequency communication needs to adapt the multi-channel parallel transmission, including three aspects: the research of communication mode, the research of data transmission frame format and the study of error correction coding as shown in Figure 1. 


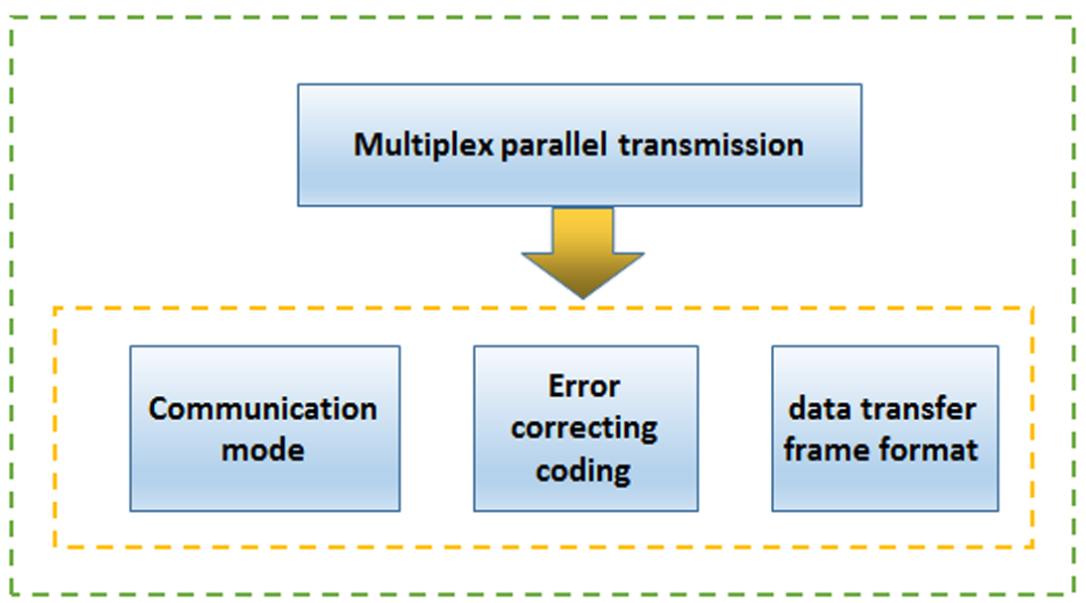

Figure 1. The research model.

\subsection{Communication model}

The traditional frequency communication system usually adopts master-slave communication model. In this model, the user terminal must receive sub-site broadcast and then send the ACK information. Meanwhile, each downlink data indicated by two position of voltage distortion signal of the two frequency cycle, and each uplink information indicated by the four frequency cycle current distortion signal position. Assume $T$ denotes the frequency cycle, the downlink data occupies the M-bit, the uplink data for the N-bit, the data structure is shown as Figure 2.

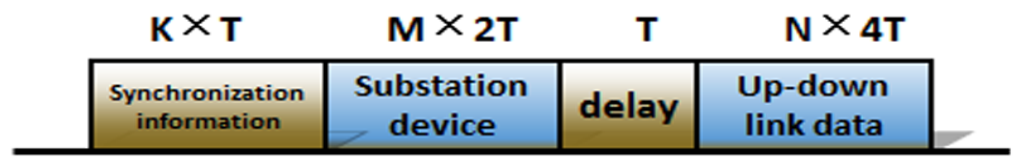

Figure 2. The data structure in the master-slave communication model.

However, there are some weaknesses in this model. When the fault occurs, the communication terminal can only wait for the sub-station inspection to be able to upload alarm information. And due to the low frequency of communication frequency, sub-station equipment inspection time is too long for the inspections of all user terminals, which is very difficult to obtain real-time fault alarm information. Therefore, we design a novel model: the active uplink communication model shown as figure 3. In this model, we proposed a positive uplink communication as a supplement, when the terminal detects an electrical fault, it can send the alarm to the sub-station information actively.

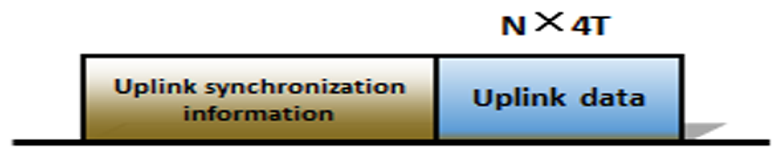

Figure 3. The data structure in the active uplink communication model.

In the active model, the uplink synchronization information provides the basis for determining the start time of the data encoding for the sub-station device, and the uplink data encoding is consistent with the master-slave mode. 


\subsection{Transmission frame}

The uplink synchronization information is modulated by pseudo-random coding combined with Manchester coding. And the sub-station device determines the starting time of the uplink data by signal synthesis and time-frequency analysis, and determines the modulation time domain of the signal, so that the burst of the uplink alarm data. Thus, we present a new downlink frequency communication frame format as shown in Figure 4.

\begin{tabular}{|l|l|c|c|c|c|c|}
\hline Synchronize & Frame Header & $\begin{array}{c}\text { Address } \\
\text { information }\end{array}$ & $\begin{array}{c}\text { Function } \\
\text { code }\end{array}$ & $\begin{array}{c}\text { Control } \\
\text { domain }\end{array}$ & $\begin{array}{c}\text { Error } \\
\text { correction }\end{array}$ & Frame tail \\
\hline
\end{tabular}

Figure 4. The downlink frequency communication frame format.

As shown in Figure 4, the synchronization information is the basis for the signal detection of the physical layer. The address information reflects the location of the user terminal in the distribution network. The control domain information may have control type, parameter setting and so on. The error correction coding can reduce the system SNR demand. Down the contents of the instructions more simple, you can use a fixed frame length mode.

As a result of multi-channel frequency communication parallel transmission, address information, including: $10 \mathrm{KV}$ branch number, distribution transformer number, phase number, low side user number. According to the same substation $10 \mathrm{KV}$ branch number, in the same branch on the distribution of the number of phase number 3 (A, B, C), each distribution single-phase power supply terminal number of features, address information using 2 to 3 words Section is suitable.

In the functional code, control domain, should meet the functional requirements of the case, as short as possible. In the case of error correction coding, the coding gain and the coding efficiency should be balanced.

The structure of the upstream coding is similar to the downstream coding as shown in figure 5 . The difference is that the response data field is long and the situation is complicated. Therefore, the variable frame length is used. The frame length information needs to be included in the function code, and the error correction coding needs to be treated differently.

When the frequency communication technology is used in FTU, meter reading terminal information transmission, it also need those areas of communication protocol adapter, in particular, to consider the frequency of communication frequency situation.

\begin{tabular}{|c|c|c|c|c|c|c|}
\hline Leading & Frame Header & $\begin{array}{c}\text { Address } \\
\text { information }\end{array}$ & $\begin{array}{c}\text { Function } \\
\text { code }\end{array}$ & Data domain & $\begin{array}{c}\text { Error } \\
\text { correction }\end{array}$ & Frame tail \\
\hline
\end{tabular}

Figure 5. The uplink frequency communication frame format.

\subsection{Error correction}

According to the research situation of the research group, the downlink frequency communication data adopts the fixed frame length, and the $\mathrm{BCH}$ error correction coding can get the better effect, and the uplink and the traffic frequency communication data can be used to improve the communication performance. Power frequency data is using RS error correction coding more appropriate. In this group has been carried out in the transmission test, the use of error correction coding, the uplink and downlink communication data success rate can be improved.

In the case of three-phase transmission combined with single-phase multiplexing, the interference noise will be further serious, so there are

It is necessary to strengthen research in this area. 


\subsection{Influence of voltage phase difference on power frequency communication}

Due to the presence of the voltage phase difference between the transmission and output terminals of the power frequency communication signal, the traditional signal demodulation method based on the local voltage zero crossing will appear in the time domain deviation. In the industrial distribution network, due to the large reactive power in the line current, The phase difference is large, which will cause serious impact on the signal demodulation, the subject of cross-correlation demodulation of the next line of data as an example to analyze the impact of the situation.

In the voltage signal sense output, the noise has a large randomness, and for the sake of convenience, we assume the noise is the Gaussian white noise with zero mean. The reference signal and noise related output $N(\lambda)$, the mean and variance are as shown in $\operatorname{Eq}(1)$ :

$$
\begin{gathered}
E[N(\lambda)]=0 \\
\delta_{\mathrm{n}}^{2}=E\left[N^{2}(\lambda)\right]=\frac{K^{2}}{2} N_{0} E_{s}
\end{gathered}
$$

Where $N_{0}$ indicates Noise power. $\mathrm{Eq}(1)$ shows the noise variance $\sigma_{\mathrm{n}}{ }^{2}$ is not affected by the correlation delay.

\section{Applications of new power frequency communication technology}

Power frequency communication technology utilizes the weak low frequency distortion signal $(200 \mathrm{~Hz}$ to $600 \mathrm{~Hz}$ ) of the voltage and current waveform in the power grid to realize the information transmission. Because the distortion signal can cross the transformer, the power frequency communication technology has the unique advantage that can cross the transformer long distance communication; In the field of electricity information collection, user side intelligent power equipment remote control, distributed generation island detection and other fields

\subsection{Electricity information collection}

Remote automatic meter reading technology AMR is a no need to reach the first check function can complete the automatic meter reading of the new meter reading. It is through the public telephone network, load control channel or low-voltage distribution line carrier and other communication links, the power of the data automatically transmitted to the computer power management center for processing. The meter reading scheme based on power line frequency communication technology is shown in Figure 6.

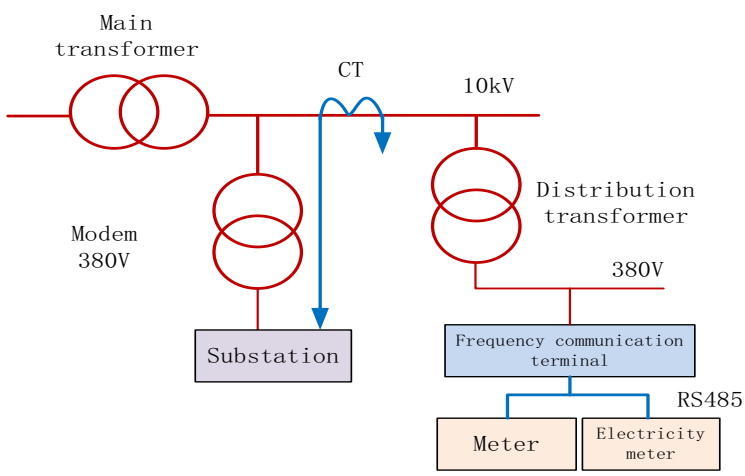

Figure 6. Remote meter reading based on power frequency communication. 
After the electricity information is sent through the frequency communication technology to the substation, the internal enterprise networks forward these data to the management through the power system.

When using multi-channel frequency communication signal transmission technology, the singlephase user's downlink rate of $25 \mathrm{~b} / \mathrm{s}$, the uplink rate up to $75 \mathrm{~b} / \mathrm{s}$, lower than the current GPRS with PLC way, but the power line frequency communication technology has the ability to cross Transformer, no need to install the coupling device in the $10 \mathrm{KV}$ line characteristics, you can in the distribution of small number of users and scattered areas, such as rural areas or grassland on the longrange meter reading.

\subsection{User side intelligent power equipment remote control}

Intelligent electricity is an important part of building a strong smart grid, which means the use of advanced measurement, efficient control, high-speed communications, fast energy storage and other technologies to achieve rapid market response, fair and accurate measurement, real-time data collection, Efficient and convenient to build a grid and customer energy flow, information flow, realtime interactive business flow for the new relationship.

In the smart electricity, in particular, emphasizing the exchange of information between smart meters and power grid, in order to achieve intelligent power control, based on power line frequency communication technology user side intelligent power remote control as shown in Figure 7.

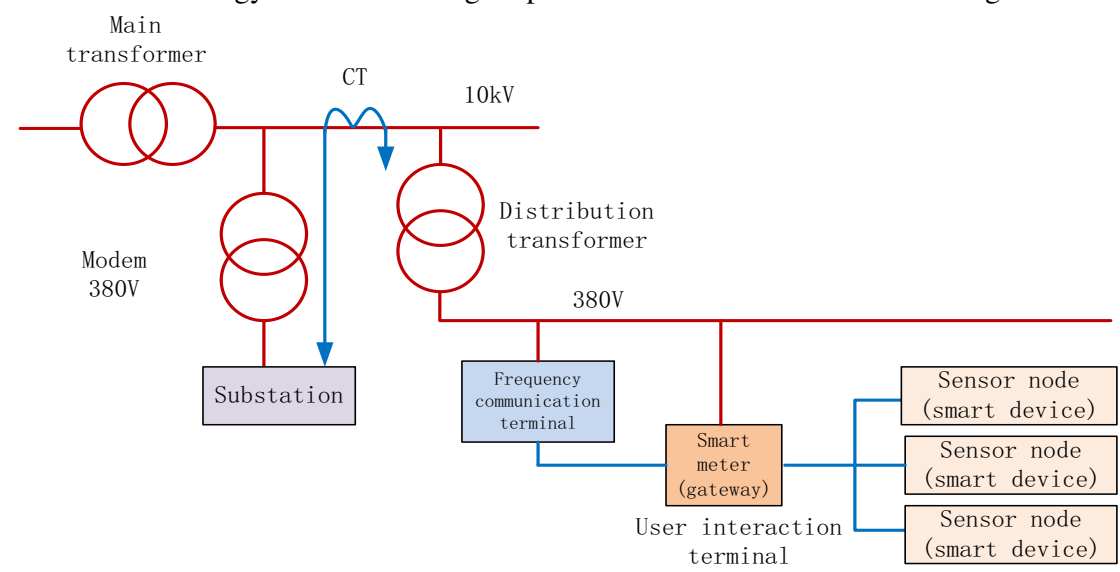

Figure7. Remote control of intelligent power consumption for power line based on power line communication

In the structure of Figure 7, according to the characteristics of frequency communication, most of the processing in the local, the key data upload, for the location of the three-phase users scattered, the downlink rate of $75 \mathrm{~b} / \mathrm{s}$, the maximum uplink rate of up $225 \mathrm{~b} / \mathrm{s}$, although the rate is low, the power line frequency communication technology does not need to install the coupling equipment in the $10 \mathrm{KV}$ line, and there is no communication operation cost, in the local processing to reduce the amount of transmission information, the way has a good price.

\section{Conclusion}

Power Line Carrier (PLC) communication and wireless communication are important communication means in the distribution communication network. The power line is susceptible to the influence of line load and interference. In this paper, we focus on the frequency communication technology in power distribution communication network, and design a novel technology in communication mode, 
error correcting coding and data transfer frame format. We also discuss the influence of voltage phase difference on power frequency communication. Power line frequency communication is in the zero to add a weak distortion to achieve the signal modulation. The research of the new power line frequency communication technology includes multi-feeder, three-phase, multi-channel signal transmission model research, multi-channel frequency signal modulation and demodulation technology research, communication protocol research, etc. Meanwhile, we present the application scenario Electricity information collection, electricity remote control and other power business in smart grid with the novel frequency communication technology.

\section{References}

1. Yu Q, Huang $\mathrm{H}, \mathrm{Yu}$ Y, et al. Research on Heterogeneous Communication Network for Power Distribution Automation, International Conference on Material, Mechanical and Manufacturing Engineering. 2015.

2. Razani M. Power Line Carrier Communication. Journal of Chongqing Institute of Technology, 2011.

3. Varadarajan B, Kim I, Dabak A, et al. Empirical measurements of the low-frequency power-line communications channel in rural North America, IEEE International Symposium on Power Line Communications and ITS Applications. IEEE, 2011:463-467.

4. Chervenkov A, Chervenkova T. Investigation of the Influence of the Electromagnetic Field in the Vicinity of High Voltage Overhead Line[C]// Materials Science Forum. 2016:190-195.

5. Wu F, Li X, Duan J. Improved Elimination Scheme of Current Zero-Crossing Distortion in Unipolar Hysteresis Current Controlled Grid-Connected Inverter. Industrial Informatics IEEE Transactions on, 2015, 11(5):1111-1118.

6. López-Colino F, Sanchez A, Castro A D, et al. Handling input voltage frequency variations in power factor correctors with pre-calculated duty cycles. Electrical Engineering, 2016:1-12.

7. Facina M S P, Ribeiro M V. The influence of transmission power and frequency bandwidth on inhome cooperative power line communication, International Symposium on Power Line Communications and ITS Applications. IEEE, 2015:239-244.

8. Ebenezer M, Ramachandralal R M. Study and Analysis of the Effect of Harmonics on the Hot Spot Temperature of a Distribution Transformer Using Finite-Volume Method. Electric Power Components \& Systems, 2015, 43(20):1-11.

9. Yong L I, Zuo H U. Fault analysis of Dy11 connection mode of distribution transformers parallel. Power System Protection \& Control, 2009.

10. Jian-Qi L I, Huang B Y, Wang Z H. Application of power frequency communication technology in power distribution network. Telecommunications for Electric Power System, 2009.

11. Mastellone S, Lee D, Spong M W. Master-slave synchronization with switching communication through passive model-based control design, American Control Conference. IEEE Xplore, 2006:6 pp.

12. Tujkovic D. Mobile subscriber information transmission over multiple uplink frames. 2016. 